\title{
A Study of Nonlinear Fractional $q$-Difference Equations with Nonlocal Integral Boundary Conditions
}

\author{
Ahmed Alsaedi, Bashir Ahmad, and Hana Al-Hutami \\ Department of Mathematics, Faculty of Science, King Abdulaziz University, P.O. Box 80203, Jeddah 21589, Saudi Arabia \\ Correspondence should be addressed to Ahmed Alsaedi; aalsaedi@hotmail.com
}

Received 12 July 2013; Revised 9 September 2013; Accepted 9 September 2013

Academic Editor: Dumitru Baleanu

Copyright (C) 2013 Ahmed Alsaedi et al. This is an open access article distributed under the Creative Commons Attribution License, which permits unrestricted use, distribution, and reproduction in any medium, provided the original work is properly cited.

\begin{abstract}
This paper is concerned with the existence and uniqueness of solutions for a boundary value problem of nonlinear fractional $q$ difference equations with nonlocal integral boundary conditions. The existence results are obtained by applying some well-known fixed point theorems and illustrated with examples.
\end{abstract}

\section{Introduction}

Several kinds of boundary value problems of fractionalorder have recently been investigated by many researchers. Fractional derivatives appear naturally in the mathematical modelling of dynamical systems involving fractals and chaos. In fact, the concept of fractional calculus has played a key role in improving the work based on integer-order (classical) calculus in several diverse disciplines of science and engineering. This might have been due to the fact that fractionaldifferential operators help to understand the hereditary phenomena in many materials and processes in a better way than the corresponding integer-order differential operators. Examples include physics, chemistry, biology, biophysics, blood flow phenomena, control theory, signal and image processing, and economics [1-4]. For some recent results on the topic, see a series of papers [5-12] and the references therein.

Fractional $q$-difference equations, regarded as fractional analogue of $q$-difference equations, have been studied by several researchers. For some earlier work on the subject, we refer to $[13,14]$, whereas the recent development on the existence theory of fractional $q$-difference equations can be found in [15-25]. In a recent paper [26], the authors investigated a nonlocal boundary value problem of nonlinear fractional $q$-difference equations:

$$
{ }^{c} D_{q}^{\alpha} x(t)=f(t, x(t)), \quad 0 \leq t \leq 1,1<\alpha \leq 2,
$$

$$
\begin{aligned}
& \alpha_{1} x(0)-\beta_{1} D_{q} x(0)=\gamma_{1} x\left(\eta_{1}\right), \\
& \alpha_{2} x(1)+\beta_{2} D_{q} x(1)=\gamma_{2} x\left(\eta_{2}\right),
\end{aligned}
$$

where $f \in C([0,1] \times \mathbb{R}, \mathbb{R}),{ }^{c} D_{q}^{\alpha}$ is the fractional $q$-derivative of the Caputo type, $D_{q}$ is $q$-derivative, and $\alpha_{i}, \beta_{i}, \gamma_{i} \in \mathbb{R}, \eta_{i} \in$ $(0,1), i=1,2$.

The purpose of the present paper is to study the following nonlocal boundary value problem of nonlinear fractional $q$ difference equations:

$$
\begin{gathered}
{ }^{c} D_{q}^{\beta} x(t)+\lambda x(t)=f(t, x(t)), \\
t \in[0,1], 1<\beta \leq 2,0<q<1, \\
x(0)=0, \quad x(1)=I_{q} x(\eta)=\int_{0}^{\eta} x(s) d_{q} s, \quad 0<\eta<1,
\end{gathered}
$$

where $f \in C([0,1] \times \mathbb{R}, \mathbb{R}),{ }^{c} D_{q}^{\beta}$ is the fractional $q$-derivative of the Caputo type, and $\lambda$ is a real number.

The paper is organized as follows. Section 2 contains some necessary background material on the topic, while the main results are presented in Section 3. We make use of Banach's contraction principle, Krasnoselskii's fixed point theorem, and Leray-Schauder nonlinear alternative to establish the existence results for the problem at hand. 


\section{Preliminaries on Fractional $q$-Calculus}

Here we recall some definitions and fundamental results on fractional $q$-calculus.

Definition 1 (see [13]). Let $\beta \geq 0$, let $0<q<1$, and let $f$ be a function defined on $[0,1]$. The fractional $q$-integral of the Riemann-Liouville type is $\left(I_{q}^{0} f\right)(t)=f(t)$ and

$$
\left(I_{q}^{\beta} f\right)(t)=\int_{0}^{t} \frac{(t-q s)^{(\beta-1)}}{\Gamma_{q}(\beta)} f(s) d_{q}(s), \quad \beta>0, t \in[0,1],
$$

where

$$
\Gamma_{q}(\beta)=\frac{(1-q)^{(\beta-1)}}{(1-q)^{\beta-1}}, \quad 0<q<1 .
$$

Recall that $\Gamma_{q}(\beta+1)=\lceil\beta\rceil_{q} \Gamma_{q}(\beta)$, with

$$
\begin{gathered}
\lceil\beta\rceil_{q}=\frac{q^{\beta}-1}{q-1}, \quad(1-q)^{(0)}=1, \\
(1-q)^{(n)}=\prod_{k=0}^{n-1}\left(1-q^{k+1}\right), \quad n \in \mathbb{N} .
\end{gathered}
$$

More generally, if $\beta \in \mathbb{R}$, then

$$
(1-q)^{(\beta)}=\prod_{i=0}^{\infty} \frac{\left(1-q^{i+1}\right)}{\left(1-q^{\beta+i+1}\right)} .
$$

Lemma 2 (see [27]). For $\beta \in \mathbb{R}^{+}, \lambda \in(-1, \infty)$, the following is valid:

$$
I_{q}^{\beta}\left((t-a)^{(\lambda)}\right)=\frac{\Gamma_{q}(\lambda+1)}{\Gamma_{q}(\beta+\lambda+1)}(t-a)^{(\beta+\lambda)}, \quad 0<a<t<b .
$$

In particular, for $\lambda=0, a=0$, using $q$-integration by parts, we have

$$
\begin{aligned}
\left(I_{q}^{\beta} 1\right)(t) & =\frac{1}{\Gamma_{q}(\beta)} \int_{0}^{t}(t-q s)^{(\beta-1)} d_{q} s \\
& =\frac{1}{\Gamma_{q}(\beta)} \int_{0}^{t} \frac{D_{q}\left((t-s)^{(\beta)}\right)}{-[\beta]_{q}} d_{q} s \\
& =\frac{-1}{\Gamma_{q}(\beta+1)} \int_{0}^{t} D_{q}\left((t-s)^{(\beta)}\right) d_{q} s \\
& =\frac{1}{\Gamma_{q}(\beta+1)} t^{(\beta)} .
\end{aligned}
$$

For $0<q<1$, we define the $q$-derivative of a real valued function $f$ as

$$
\begin{gathered}
D_{q} f(t)=\frac{f(t)-f(q t)}{(1-q) t}, \quad t \neq 0, \\
D_{q} f(0)=\lim _{n \rightarrow \infty} \frac{f\left(s q^{n}\right)-f(0)}{s q^{n}}, \quad s \neq 0 .
\end{gathered}
$$

For more details, see [28].
Definition 3 (see [29]). The fractional $q$-derivative of the Riemann-Liouville type of order $\beta \geq 0$ is defined by $\left(D_{q}^{0} f\right)(t)=f(t)$ and

$$
\left(D_{q}^{\beta} f\right)(t)=\left(D_{q}^{[\beta]} I_{q}^{[\beta]-\beta} f\right)(t), \quad \beta>0,
$$

where $[\beta]$ is the smallest integer greater than or equal to $\beta$.

Definition 4 (see [29]). The fractional $q$-derivative of the Caputo type of order $\beta \geq 0$ is defined by

$$
\left({ }^{c} D_{q}^{\beta} f\right)(t)=\left(I_{q}^{[\beta]-\beta} D_{q}^{[\beta]} f\right)(t), \quad \beta>0,
$$

where $[\beta]$ is the smallest integer greater than or equal to $\beta$.

Now we state some known results involving $q$-derivatives and $q$-integrals.

Lemma 5 (see [29]). Let $\beta, \gamma \geq 0$, and let $f$ be a function defined on $[0,1]$. Then

$$
\begin{aligned}
& \text { (i) }\left(I_{q}^{\beta} I_{q}^{\gamma} f\right)(t)=\left(I_{q}^{\beta+\gamma} f\right)(t), \\
& \text { (ii) }\left(D_{q}^{\beta} I_{q}^{\beta} f\right)(t)=f(t), \\
& \text { (iii) }\left(I_{q}^{\beta c} D_{q}^{\beta} f\right)(t)=f(t)-\sum_{k=0}^{[\beta]-1}\left(t^{k} / \Gamma_{q}(k+1)\right)\left({ }^{c} D_{q}^{k} f\right)(0),
\end{aligned}
$$
$\beta>0$.

Lemma 6 (see [19]). Let $\beta \geq 0$ and $n \in \mathbb{N}$. Then, the following equality holds:

$$
\left(I_{q}^{\beta} D_{q}^{n} f\right)(t)=D_{q}^{n} I_{q}^{\beta} f(t)-\sum_{k=0}^{[\beta]-1} \frac{t^{\beta-n+k}}{\Gamma_{q}(\beta-n+k)}\left(D_{q}^{k} f\right)(0) .
$$

In the forthcoming analysis, the following lemma plays a pivotal role.

Lemma 7. Let $h \in C([0,1], \mathbb{R})$ be a given function. Then the unique solution of the boundary value problem,

$$
\begin{gathered}
{ }^{c} D_{q}^{\beta} x(t)+\lambda x(t)=h(t), \\
t \in[0,1], \quad 1<\beta \leq 2,0<q<1, \\
x(0)=0, \quad x(1)=\int_{0}^{\eta} x(s) d_{q} s, \quad 0<\eta<1,
\end{gathered}
$$

is given by

$$
\begin{aligned}
x(t)= & \int_{0}^{t} \frac{(t-q s)^{(\beta-1)}}{\Gamma_{q}(\beta)}(h(s)-\lambda x(s)) d_{q} s \\
& +\delta_{1} t \int_{0}^{\eta} \frac{(\eta-q s)^{(\beta)}}{\Gamma_{q}(\beta+1)}(h(s)-\lambda x(s)) d_{q} s \\
& -\delta_{1} t \int_{0}^{1} \frac{(1-q s)^{(\beta-1)}}{\Gamma_{q}(\beta)}(h(s)-\lambda x(s)) d_{q} s,
\end{aligned}
$$

where

$$
\delta_{1}=\frac{1+q}{1+q-\eta^{2}} .
$$


Proof. Using Lemma 5, we can write the solution of fractional $q$-difference equation in (13) as

$$
\begin{aligned}
x(t)= & \int_{0}^{t} \frac{(t-q s)^{(\beta-1)}}{\Gamma_{q}(\beta)}(h(s)-\lambda x(s)) d_{q} s \\
& +c_{0} t+c_{1}, \quad t \in[0,1] .
\end{aligned}
$$
0 and

Using the boundary conditions of (13) in (16), we get $c_{1}=$

$$
\begin{aligned}
c_{0}=\delta_{1} & {\left[\int_{0}^{\eta} \frac{(\eta-q s)^{(\beta)}}{\Gamma_{q}(\beta+1)}(h(s)-\lambda x(s)) d_{q} s\right.} \\
& \left.-\int_{0}^{1} \frac{(1-q s)^{(\beta-1)}}{\Gamma_{q}(\beta)}(h(s)-\lambda x(s)) d_{q} s\right],
\end{aligned}
$$

where $\delta_{1}$ is given by (15). Substituting the values of $c_{0}, c_{1}$ in (16), we obtain (14).

Let $\mathscr{C}:=C([0,1], \mathbb{R})$ denote the Banach space of all continuous functions from $[0,1]$ to $\mathbb{R}$ endowed with the norm defined by $\|x\|=\sup \{|x(t)|: t \in[0,1]\}$.

By virtue of Lemma 7, we define an operator $\mathscr{G}: \mathscr{C} \rightarrow \mathscr{C}$ as

$$
\begin{aligned}
(\mathscr{G} x)(t)= & \int_{0}^{t} \frac{(t-q s)^{(\beta-1)}}{\Gamma_{q}(\beta)}(f(s, x(s))-\lambda x(s)) d_{q} s \\
& +\delta_{1} t \int_{0}^{\eta} \frac{(\eta-q s)^{(\beta)}}{\Gamma_{q}(\beta+1)}(f(s, x(s))-\lambda x(s)) d_{q} s \\
& -\delta_{1} t \int_{0}^{1} \frac{(1-q s)^{(\beta-1)}}{\Gamma_{q}(\beta)}(f(s, x(s))-\lambda x(s)) d_{q} s
\end{aligned}
$$

and note that the given problem (2) has solutions only if the operator equation $\mathscr{G} x=x$ has fixed points.

\section{Main Results}

In the sequel, we assume that

$\left(A_{1}\right) f:[0,1] \times \mathbb{R} \rightarrow \mathbb{R}$ is a continuous function and that there exists a $q$-integrable function $\zeta:[0,1] \rightarrow \mathbb{R}$ such that $|f(t, x)-f(t, y)| \leq \zeta(t)|x-y|, t \in[0,1], x$, $y \in \mathbb{R}$. tions:

For computational convenience, we introduce the nota-

$$
\Lambda=\mu_{1}+|\lambda| \mu_{2}
$$

where

$$
\begin{aligned}
\mu_{1} & :=\left(1+\left|\delta_{1}\right|\right)\left(I_{q}^{\beta} \zeta\right)(1)+\left|\delta_{1}\right|\left(I_{q}^{\beta+1} \zeta\right)(\eta), \\
\mu_{2} & :=\left(\frac{1}{\Gamma_{q}(\beta+1)}\left(1+\left|\delta_{1}\right|\right)+\frac{\left|\delta_{1}\right| \eta^{\beta+1}}{\Gamma_{q}(\beta+2)}\right) .
\end{aligned}
$$

Our first existence result is based on Leray-Schauder nonlinear alternative.
Lemma 8 (nonlinear alternative for single valued maps [30]). Let $E$ be a Banach space, $C$ a closed, convex subset of $E$, and $V$ an open subset of $C$ with $0 \in V$. Suppose that $\mathscr{G}: \bar{V} \rightarrow C$ is a continuous, compact (i.e., $\mathscr{G}(\bar{V})$ is a relatively compact subset ofC) map. Then either

(i) $\mathscr{G}$ has a fixed point in $\bar{V}$, or

(ii) there is $x \in \partial V$ (the boundary of $V$ in $C$ ) and $\varepsilon \in(0,1)$ with $x=\varepsilon \mathscr{G}(x)$.

Theorem 9. Suppose that $f:[0,1] \times \mathbb{R} \rightarrow \mathbb{R}$ is a continuous function. In addition, it is assumed that

$\left(A_{2}\right)$ there exist functions $h_{1}, h_{2} \in L^{1}\left([0,1], \mathbb{R}^{+}\right)$and $a$ nondecreasing function $\vartheta: \mathbb{R}^{+} \rightarrow \mathbb{R}^{+}$such that $|f(t, x)| \leq h_{1}(t) \vartheta(|x|)+h_{2}(t)$, for $(t, x) \in[0,1] \times \mathbb{R}$.

Then the problem (2) has at least one solution on $[0,1]$ if there exists a positive number $\omega$ such that

$$
\omega>\frac{\vartheta(\omega) \nu_{1}+\nu_{2}}{1-|\lambda| \mu_{2}}
$$

where

$$
\begin{gathered}
|\lambda| \neq \frac{1}{\mu_{2}} \\
v_{i}:=\left(1+\left|\delta_{1}\right|\right)\left(I_{q}^{\beta} h_{i}\right)(1)+\left|\delta_{1}\right|\left(I_{q}^{\beta+1} h_{i}\right)(\eta), \quad i=1,2 .
\end{gathered}
$$

Proof. As a first step, it will be shown that the operator $\mathscr{G}$ : $\mathscr{C} \rightarrow \mathscr{C}$ (defined by (18)) maps bounded sets into bounded sets in $C([0,1], \mathbb{R})$. Notice that $\mathscr{G}$ is continuous. For a positive number $\sigma$, let $\bar{B}_{\sigma}=\{x \in C([0,1], \mathbb{R}):\|x\| \leq \sigma\}$ be a bounded set in $C([0,1], \mathbb{R})$. Then, for any $x \in \bar{B}_{\sigma}$, we find that

$|(\mathscr{G} x)(t)|$

$$
\begin{aligned}
& \leq \int_{0}^{t} \frac{(t-q s)^{(\beta-1)}}{\Gamma_{q}(\beta)}(|f(s, x(s))|+|\lambda||x(s)|) d_{q} s \\
& \quad+\left|\delta_{1}\right||t| \int_{0}^{\eta} \frac{(\eta-q s)^{(\beta)}}{\Gamma_{q}(\beta+1)}(|f(s, x(s))|+|\lambda||x(s)|) d_{q} s \\
& +\left|\delta_{1}\right||t| \int_{0}^{1} \frac{(1-q s)^{(\beta-1)}}{\Gamma_{q}(\beta)}(|f(s, x(s))|+|\lambda||x(s)|) d_{q} s \\
& \leq \int_{0}^{t} \frac{(t-q s)^{(\beta-1)}\left[h_{1}(s) \vartheta(\|x\|)+h_{2}(s)\right] d_{q} s}{\Gamma_{q}(\beta)}\left[h_{1}(s) \vartheta(\|x\|)+h_{2}(s)\right] d_{q} s \\
& +\left|\delta_{1}\right||t| \int_{0}^{\eta} \frac{(\eta-q s)^{(\beta)}}{\Gamma_{q}(\beta+1)}\left[h_{1}(s) \vartheta(\|x\|)+h_{2}(s)\right] d_{q} s
\end{aligned}
$$




$$
\begin{aligned}
& \leq \vartheta(\sigma)\left\{\int_{0}^{1} \frac{(1-q s)^{(\beta-1)}}{\Gamma_{q}(\beta)} h_{1}(s) d_{q} s\right. \\
& +\left|\delta_{1}\right| \int_{0}^{\eta} \frac{(\eta-q s)^{(\beta)}}{\Gamma_{q}(\beta+1)} h_{1}(s) d_{q} s \\
& +\left\{\int_{0}^{1} \frac{(1-q s)^{(\beta-1)}}{\Gamma_{q}(\beta)} h_{2}(s) d_{q} s\right. \\
& \left.+\left|\delta_{1}\right| \int_{0}^{1} \frac{(1-q s)^{(\beta-1)}}{\Gamma_{q}(\beta)} h_{1}(s) d_{q} s\right\} \\
& +\mid \int_{0}^{\eta} \frac{(\eta-q s)^{(\beta)}}{\Gamma_{q}(\beta+1)} h_{2}(s) d_{q} s \\
& +\sigma|\lambda|\left\{\int_{0}^{1} \frac{(1-q s)^{(\beta-1)}}{\Gamma_{q}(\beta)} d_{q} s+\left|\delta_{1}\right| \int_{0}^{1} \frac{(1-q s)^{(\beta-1)}}{\Gamma_{q}(\beta)} h_{2}(s) d_{q} s\right\} \\
& \left.+\left|\delta_{1}\right| \int_{0}^{1} \frac{(1-q s)^{(\beta-1)}}{\Gamma_{q}(\beta)} d_{q} s\right\} \\
& +(\beta)
\end{aligned}
$$$$
\leq \vartheta(\sigma) \nu_{1}+\nu_{2}+\sigma|\lambda| \mu_{2}
$$

This establishes our assertion.

Next, we show that the operator $\mathscr{G}$ maps bounded sets into equicontinuous sets of $C([0,1], \mathbb{R})$. Taking $t_{1}, t_{2} \in[0,1]$ with $t_{1}<t_{2}$ and $x \in \bar{B}_{\sigma}$ together with the inequality: $\left(t_{2}-q s\right)^{(\beta-1)}-\left(t_{1}-q s\right)^{(\beta-1)} \leq\left(t_{2}-t_{1}\right)$ for $0<t_{1}<t_{2}$ (see [19] p. 4), we obtain

$$
\begin{aligned}
& \left|(\mathscr{G} x)\left(t_{2}\right)-(\mathscr{G} x)\left(t_{1}\right)\right| \\
& \leq \mid \int_{0}^{t_{2}} \frac{\left(t_{2}-q s\right)^{(\beta-1)}}{\Gamma_{q}(\beta)}(f(s, x(s))-\lambda x(s)) d_{q} s \\
& \quad-\int_{0}^{t_{1}} \frac{\left(t_{1}-q s\right)^{(\beta-1)}}{\Gamma_{q}(\beta)}(f(s, x(s))-\lambda x(s)) d_{q} s \mid \\
& +\left|\delta_{1}\right|\left(t_{2}-t_{1}\right) \int_{0}^{\eta} \frac{(\eta-q s)^{(\beta)}}{\Gamma_{q}(\beta+1)} \\
& \quad \times(|f(s, x(s))|+|\lambda||x(s)|) d_{q} s . \\
& +\left|\delta_{1}\right|\left(t_{2}-t_{1}\right) \int_{0}^{1} \frac{(1-q s)^{(\beta-1)}}{\Gamma_{q}(\beta)} \\
& \times(|f(s, x(s))|+|\lambda||x(s)|) d_{q} s .
\end{aligned}
$$

$$
\begin{aligned}
& \leq \mid \int_{0}^{t_{1}} \frac{\left(t_{2}-t_{1}\right)}{\Gamma_{q}(\beta)}\left[h_{1}(s) \vartheta(\sigma)+h_{2}(s)+|\lambda| \sigma\right] d_{q} s \\
& +\int_{t_{1}}^{t_{2}} \frac{\left(t_{2}-q s\right)^{(\beta-1)}}{\Gamma_{q}(\beta)}\left[h_{1}(s) \vartheta(\sigma)+h_{2}(s)+|\lambda| \sigma\right] d_{q} s \mid \\
& +\left|\delta_{1}\right|\left(t_{2}-t_{1}\right) \int_{0}^{\eta} \frac{(\eta-q s)^{(\beta)}}{\Gamma_{q}(\beta+1)} \\
& \times\left[h_{1}(s) \vartheta(\sigma)+h_{2}(s)+|\lambda| \sigma\right] d_{q} s . \\
& +\left|\delta_{1}\right|\left(t_{2}-t_{1}\right) \int_{0}^{1} \frac{(1-q s)^{(\beta-1)}}{\Gamma_{q}(\beta)} \\
& \times\left[h_{1}(s) \vartheta(\sigma)+h_{2}(s)+|\lambda| \sigma\right] d_{q} s .
\end{aligned}
$$

It is obvious that the right hand side of the above inequality tends to zero independently of $x \in \bar{B}_{\sigma}$ as $t_{2} \rightarrow t_{1}$. Therefore the operator $\mathscr{G}$ is completely continuous by the Arzelá-Ascoli theorem.

Thus the operator $\mathscr{G}$ satisfies the hypothesis of Lemma 8 and hence by its conclusion, either condition (i) or condition (ii) holds. We claim that the conclusion (ii) is not possible.

Let $V=\{x \in C([0,1], \mathbb{R}):\|x\|<\omega\}$ with $\omega$ given by $(21)$. Then we will show that $\|\mathscr{G} x\|<\omega$. Indeed, by means of $\left(A_{4}\right)$, we get

$\|\mathscr{G} x\|$

$$
\begin{aligned}
& \leq \mathcal{\vartheta}(\|x\|)\left\{\int_{0}^{1} \frac{(1-q s)^{(\beta-1)}}{\Gamma_{q}(\beta)} h_{1}(s) d_{q} s\right. \\
& +\left|\delta_{1}\right| \int_{0}^{\eta} \frac{(\eta-q s)^{(\beta)}}{\Gamma_{q}(\beta+1)} h_{1}(s) d_{q} s \\
& +\left\{\int_{0}^{1} \frac{\left.(1-q s)^{(\beta-1)} \frac{(1-q s)^{(\beta-1)}}{\Gamma_{q}(\beta)} h_{1}(s) d_{q} s\right\}}{\Gamma_{q}(\beta)} h_{2}(s) d_{q} s\right. \\
& +\left|\delta_{1}\right| \int_{0}^{\eta} \frac{(\eta-q s)^{(\beta)}}{\Gamma_{q}(\beta+1)} h_{2}(s) d_{q} s \\
& \left.+\left|\delta_{1}\right| \int_{0}^{1} \frac{(1-q s)^{(\beta-1)}}{\Gamma_{q}(\beta)} h_{2}(s) d_{q} s\right\} \\
& +9(\omega) v_{1}+v_{2}+\omega|\lambda| \mu_{2}<\omega . \\
& +M|\lambda|\left\{\int_{0}^{1} \frac{(1-q s)^{(\beta-1)}}{\Gamma_{q}(\beta)} d_{q} s+\left|\delta_{1}\right| \int_{0}^{\eta} \frac{(\eta-q s)^{(\beta)}}{\Gamma_{q}(\beta+1)} d_{q} s\right. \\
& \left.+\delta_{1} \mid \int_{0}^{1} \frac{(1-q s)^{(\beta-1)}}{\Gamma_{q}(\beta)} d_{q}\right\}
\end{aligned}
$$


Assume that there exist $x \in \partial V$ and $\varepsilon \in(0,1)$ such that $x=$ $\varepsilon \mathscr{G} x$. Then for such a choice of $x$ and $\varepsilon$, we get a contradiction:

$$
\begin{aligned}
\omega & =\|x\|=\varepsilon\|\mathscr{G} x\|<\vartheta(\|x\|) \nu_{1}+\nu_{2}+\omega|\lambda| \mu_{2} \\
& =\vartheta(\omega) \nu_{1}+\nu_{2}+\omega|\lambda| \mu_{2}<\omega .
\end{aligned}
$$

Thus it follows by Lemma 8 that $\mathscr{G}$ has a fixed point $x \in \bar{V}$ which is a solution of the problem (2). This completes the proof.

Remark 10. In case we take $h_{1}, h_{2}$ in $\left(A_{2}\right)$ to be continuous, then $v_{i}=\mu_{2}\left\|h_{i}\right\|, i=1,2$, where $\mu_{2}$ is given by (20).

Our next result deals with existence and uniqueness of solutions for the problem (2) and is based on Banach's fixed point theorem.

Theorem 11. Suppose that the assumption $\left(A_{1}\right)$ holds and that $\Lambda<1$, where $\Lambda$ is given by (19). Then the boundary value problem (2) has a unique solution.

Proof. Fix $N=\sup _{t \in[0,1]}|f(t, 0)|<\infty, r \geq\left(N \mu_{2} / 1-\Lambda\right)$ ( $\mu_{2}$ is given by (20)), and define $\bar{B}_{r}=\{x \in \mathscr{C}:\|x\| \leq r\}$. We will show that $\mathscr{G} \bar{B}_{r} \subset \bar{B}_{r}$, where $\mathscr{G}$ is defined by (18). For $x \in \bar{B}_{r}, t \in[0,1]$, it follows by the assumption $\left(A_{1}\right)$ that

$$
\begin{aligned}
|f(t, x(t))| & \leq|f(t, x(t))-f(t, 0)|+|f(t, 0)| \\
& \leq \zeta(t)|x(t)|+|f(t, 0)| \leq \zeta(t) r+N .
\end{aligned}
$$

Then, for $x \in \bar{B}_{r}, t \in[0,1]$, we have

$$
\begin{aligned}
& |(\mathscr{G} x)(t)| \\
& \leq \int_{0}^{t} \frac{(t-q s)^{(\beta-1)}}{\Gamma_{q}(\beta)}[\zeta(s) r+N] d_{q} s \\
& +r|\lambda| \int_{0}^{t} \frac{(t-q s)^{(\beta-1)}}{\Gamma_{q}(\beta)} d_{q} s+|t|\left|\delta_{1}\right| \\
& \times \int_{0}^{\eta} \frac{(\eta-q s)^{(\beta)}}{\Gamma_{q}(\beta+1)}[\zeta(s) r+N] d_{q} s \\
& +r|\lambda| \int_{0}^{\eta} \frac{(\eta-q s)^{(\beta)}}{\Gamma_{q}(\beta+1)} d_{q} s+|t|\left|\delta_{1}\right| \\
& \times \int_{0}^{1} \frac{(1-q s)^{(\beta-1)}}{\Gamma_{q}(\beta)}[\zeta(s) r+N] d_{q} s \\
& +r|\lambda| \int_{0}^{1} \frac{(1-q s)^{(\beta-1)}}{\Gamma_{q}(\beta)} d_{q} s \\
& \leq r\left\{\int_{0}^{1} \frac{(1-q s)^{(\beta-1)}}{\Gamma_{q}(\beta)} \zeta(s) d_{q} s\right. \\
& +\left|\delta_{1}\right| \int_{0}^{\eta} \frac{(\eta-q s)^{(\beta)}}{\Gamma_{q}(\beta+1)} \zeta(s) d_{q} s \\
& \left.+\left|\delta_{1}\right| \int_{0}^{1} \frac{(1-q s)^{(\beta-1)}}{\Gamma_{q}(\beta)} \zeta(s) d_{q} s\right\}
\end{aligned}
$$

$$
\begin{aligned}
& +N\left\{\int_{0}^{1} \frac{(1-q s)^{(\beta-1)}}{\Gamma_{q}(\beta)} d_{q} s+\left|\delta_{1}\right| \int_{0}^{\eta} \frac{(\eta-q s)^{(\beta)}}{\Gamma_{q}(\beta+1)} d_{q} s\right. \\
& \left.+\left|\delta_{1}\right| \int_{0}^{1} \frac{(1-q s)^{(\beta-1)}}{\Gamma_{q}(\beta)} d_{q} s\right\} \\
& +r|\lambda|\left\{\int_{0}^{1} \frac{(1-q s)^{(\beta-1)}}{\Gamma_{q}(\beta)} d_{q} s+\left|\delta_{1}\right| \int_{0}^{\eta} \frac{(\eta-q s)}{\Gamma_{q}(\beta+1)} d_{q} s\right. \\
& +r\left\{\left(I_{q}^{\beta} \zeta\right)(1)+\left|\delta_{1}\right|\left(I_{q}^{\beta+1} \zeta\right)(\eta)+\left|\delta_{1}\right|\left(I_{q}^{\beta \zeta}\right)(1)\right\} \\
& +N\left\{\frac{1}{\Gamma_{q}(\beta+1)}+\frac{\left|\delta_{1}\right| \eta^{\beta+1}}{\Gamma_{q}(\beta+2)}+\frac{\left|\delta_{1}\right|}{\Gamma_{q}(\beta+1)}\right\} \\
& +r|\lambda|\left\{\frac{1}{\Gamma_{q}(\beta+1)}+\frac{\left|\delta_{1}\right| \eta^{\beta+1}}{\Gamma_{q}(\beta+2)}+\frac{\left|\delta_{1}\right|}{\Gamma_{q}(\beta+1)}\right\},
\end{aligned}
$$

which, in view of (19), implies that

$$
\|\mathscr{G} x\| \leq N \mu_{2}+r \Lambda \leq r .
$$

This shows that $\mathscr{G} \bar{B}_{r} \subset \bar{B}_{r}$.

Now, for $x, y \in \mathscr{C}$, we have

$$
\begin{gathered}
\|(\mathscr{G} x)-(\mathscr{G} y)\| \\
\leq \sup _{t \in[0,1]}\left\{\int_{0}^{t} \frac{(t-q s)^{(\beta-1)}}{\Gamma_{q}(\beta)}\right. \\
\times(|f(s, x(s))-f(s, y(s))| \\
+|\lambda||x(s)-y(s)|) d_{q} s \\
+\left|\delta_{1}\right||t| \int_{0}^{\eta} \frac{(\eta-q s)^{(\beta)}}{\Gamma_{q}(\beta+1)}(|f(s, x(s))-f(s, y(s))| \\
+\left|\delta_{1}\right||t| \int_{0}^{1} \frac{(1-q s)^{(\beta-1)}}{\Gamma_{q}(\beta)} \\
\quad+\left|\delta_{1}\right||t| \int_{0}^{\eta} \frac{(\eta-q s)^{(\beta)}}{\Gamma_{q}(\beta+1)} \zeta(s) d_{q} s \\
\leq\|x-y\| \sup _{t \in[0,1]}\left\{\int_{0}^{t} \frac{(t-q s)^{(\beta-1)}}{\Gamma_{q}(\beta)} \zeta(s) d_{q} s\right. \\
+|\lambda(s)|) d_{q} s
\end{gathered}
$$




$$
\begin{gathered}
+\left|\delta_{1}\right||t| \int_{0}^{1} \frac{(1-q s)^{(\beta-1)}}{\Gamma_{q}(\beta)} \zeta(s) d_{q} s \\
+|\lambda|\left(\int_{0}^{t} \frac{(t-q s)^{(\beta-1)}}{\Gamma_{q}(\beta)} d_{q} s\right. \\
+\left|\delta_{1}\right||t| \int_{0}^{\eta} \frac{(\eta-q s)^{(\beta)}}{\Gamma_{q}(\beta+1)} d_{q} s \\
\left.\left.+\left|\delta_{1}\right||t| \int_{0}^{1} \frac{(1-q s)^{(\beta-1)}}{\Gamma_{q}(\beta)} d_{q} s\right)\right\},
\end{gathered}
$$

which, by (19), takes the form

$$
\|(\mathscr{G} x)(t)-(\mathscr{G} y)(t)\| \leq \Lambda\|x-y\| .
$$

As $\Lambda<1$ (the given assumption), therefore $\mathscr{G}$ is a contraction. Hence, by Banach's contraction mapping principle, the problem (2) has a unique solution.

If we take $\zeta(t)=L$ ( $L$ is a positive constant), the condition $\Lambda<1$ becomes $L<\left(\left(1 / \mu_{2}\right)-|\lambda|\right)$ and Theorem 11 can be phrased as follows.

Corollary 12. Let there exists a constant $L \in\left(0,\left(1 / \mu_{2}\right)-|\lambda|\right)$ with $\mu_{2}$ given by (20), and let $f:[0,1] \times \mathbb{R} \rightarrow \mathbb{R}$ be a continuous function satisfying the assumption: $\mid f(t, x)-$ $f(t, y)|\leq L| x-y \mid, t \in[0,1], x, y \in \mathbb{R}$. Then the boundary value problem (2) has a unique solution.

Our last result relies on Krasnoselskii's fixed point theorem [31].

Lemma 13 (Krasnoselskii). Let $Y$ be a closed, bounded, convex, and nonempty subset of a Banach space X. Let $Q_{1}, Q_{2}$ be two operators such that (i) $Q_{1} x+Q_{2} y \in Y$ whenever $x, y \in$ $Y$; (ii) $Q_{1}$ is compact and continuous; (iii) $Q_{2}$ is a contraction mapping. Then there exists $z \in Y$ such that $z=Q_{1} z+Q_{2} z$.

Theorem 14. Let $f:[0,1] \times \mathbb{R} \rightarrow \mathbb{R}$ be a continuous function satisfying $\left(A_{1}\right)$. Furthermore, it is assumed that

$\left(A_{3}\right)$ there exist a function $\xi \in C\left([0,1], \mathbb{R}^{+}\right)$and $a$ nondecreasing function $\chi \in C\left([0,1], \mathbb{R}^{+}\right)$such that

$$
|f(t, x)| \leq \xi(t) \chi(|x|), \quad(t, x) \in[0,1] \times \mathbb{R} .
$$

If

$$
\begin{aligned}
& \left|\delta_{1}\right|\left[\int_{0}^{\eta} \frac{(\eta-q s)^{(\beta)}}{\Gamma_{q}(\beta+1)} \zeta(s) d_{q} s+\int_{0}^{1} \frac{(1-q s)^{(\beta-1)}}{\Gamma_{q}(\beta)} \zeta(s) d_{q} s\right] \\
& \quad+|\lambda|\left|\delta_{1}\right|\left[\frac{\eta^{\beta+1}}{\Gamma_{q}(\beta+2)}+\frac{1}{\Gamma_{q}(\beta+1)}\right]<1,
\end{aligned}
$$

with $|\lambda| \mu_{2}<1$, then the boundary value problem (2) has at least one solution on $[0,1]$.
Proof. Let us consider the set $\bar{B}_{\bar{r}}=\{x \in \mathscr{C}:\|x\| \leq \bar{r}\}$, where $\bar{r}$ is given by

$$
\bar{r} \geq \frac{\chi(\bar{r})\|\xi\|}{1-|\lambda| \mu_{2}}, \quad\|\xi\|=\sup _{t \in[0, t]}|\xi(t)|
$$

and introduce the operators $\mathscr{G}_{1}$ and $\mathscr{G}_{2}$ on $\bar{B}_{\bar{r}}$ as

$$
\begin{array}{r}
\left(\mathscr{G}_{1} x\right)(t)=\int_{0}^{t} \frac{(t-q s)^{(\beta-1)}}{\Gamma_{q}(\beta)}(f(s, x(s))-\lambda x(s)) d_{q} s \\
t \in[0,1]
\end{array}
$$

$$
\begin{array}{r}
\left(\mathscr{G}_{2} x\right)(t)=\delta_{1} t \int_{0}^{\eta} \frac{(\eta-q s)^{(\beta)}}{\Gamma_{q}(\beta+1)}(f(s, x(s))-\lambda x(s)) d_{q} s \\
-\delta_{1} t \int_{0}^{1} \frac{(1-q s)^{(\beta-1)}}{\Gamma_{q}(\beta)}(f(s, x(s))-\lambda x(s)) d_{q} s, \\
t \in[0,1] .
\end{array}
$$

In order to show the hypothesis of Krasnoselskii's fixed point theorem, we proceed as follows.

(i) For $x, y \in \bar{B}_{\bar{r}}$, we find that

$$
\begin{aligned}
& \left|\left(\mathscr{G}_{1} x+\mathscr{G}_{2} y\right)(t)\right| \\
& \leq \int_{0}^{t} \frac{(t-q s)^{(\beta-1)}}{\Gamma_{q}(\beta)}(\xi(s) \chi(|x(s)|)+|\lambda||x(s)|) d_{q} s \\
& \quad+\left|\delta_{1}\right| \int_{0}^{\eta} \frac{(\eta-q s)^{(\beta)}}{\Gamma_{q}(\beta+1)}(\xi(s) \chi(|y(s)|)+|\lambda||y(s)|) d_{q} s \\
& \quad+\left|\delta_{1}\right| \int_{0}^{1} \frac{(1-q s)^{(\beta-1)}}{\Gamma_{q}(\beta)}(\xi(s) \chi(|y(s)|)+|\lambda||y(s)|) d_{q} s \\
& \leq \quad \chi(\bar{r})\|\xi\|\left[\frac{1}{\Gamma_{q}(\beta+1)}\left(1+\left|\delta_{1}\right|\right)+\left|\delta_{1}\right| \frac{\eta^{\beta+1}}{\Gamma_{q}(\beta+2)}\right] \\
& \quad+\bar{r}|\lambda|\left[\frac{1}{\Gamma_{q}(\beta+1)}\left(1+\left|\delta_{1}\right|\right)+\left|\delta_{1}\right| \frac{\eta^{\beta+1}}{\Gamma_{q}(\beta+2)}\right] \\
& \leq x(\bar{r})\|\xi\| \mu_{2}+\bar{r}|\lambda| \mu_{2} \leq \bar{r} .
\end{aligned}
$$

This implies that $\mathscr{G}_{1} x+\mathscr{G}_{2} y \in \bar{B}_{\bar{r}}$.

(ii) From the continuity of $f$, it follows that the operator $\mathscr{G}_{1}$ is continuous. Also, $\mathscr{G}_{1}$ is uniformly bounded on $\bar{B}_{\bar{r}}$ as

$$
\left\|\mathscr{G}_{1} x\right\| \leq \frac{\chi(\bar{r})\|\xi\|}{\Gamma_{q}(\beta+1)}+\frac{|\lambda| \bar{r}}{\Gamma_{q}(\beta+1)} .
$$


Next, for any $x \in \bar{B}_{\bar{r}}$, and $\tau_{1}, \tau_{2} \in[0,1]$ with $\tau_{1}<\tau_{2}$, we have

$$
\begin{aligned}
& \left|\left(\mathscr{G}_{1} x\right)\left(\tau_{2}\right)-\left(\mathscr{G}_{1} x\right)\left(\tau_{1}\right)\right| \\
& =\mid \int_{0}^{\tau_{2}} \frac{\left(\tau_{2}-q s\right)^{(\beta-1)}}{\Gamma_{q}(\beta)}(f(s, x(s))-\lambda x(s)) d_{q} s \\
& \quad-\int_{0}^{\tau_{1}} \frac{\left(\tau_{1}-q s\right)^{(\beta-1)}}{\Gamma_{q}(\beta)}(f(s, x(s))-\lambda x(s)) d_{q} s \mid \\
& \leq \mid \int_{0}^{\tau_{1}} \frac{\left(\tau_{2}-q s\right)^{(\beta-1)}-\left(\tau_{1}-q s\right)^{(\beta-1)}}{\Gamma_{q}(\beta)} \\
& \quad \times(f(s, x(s))-\lambda x(s)) d_{q} s \mid \\
& +\left|\int_{\tau_{1}}^{\tau_{2}} \frac{\left(\tau_{2}-q s\right)^{(\beta-1)}}{\Gamma_{q}(\beta)}(f(s, x(s))-\lambda x(s)) d_{q} s\right| \\
& \leq \quad(\chi(\bar{r})\|\xi\|+|\lambda| \bar{r}) \\
& \quad \times\left[\int_{0}^{\tau_{1}} \frac{\left(\tau_{2}-q s\right)^{(\beta-1)}-\left(\tau_{1}-q s\right)^{(\beta-1)}}{\Gamma_{q}(\beta)} d_{q} s\right. \\
& \left.\quad+\int_{\tau_{1}}^{\tau_{2}} \frac{\left(\tau_{2}-q s\right)^{(\beta-1)}}{\Gamma_{q}(\beta)} d_{q} s\right]
\end{aligned}
$$

which is independent of $x$ and tends to zero as $\tau_{2} \rightarrow$ $\tau_{1}$. Thus, $\mathscr{G}_{1}$ is equicontinuous. So $\mathscr{G}_{1}$ is relatively compact on $\bar{B}_{\bar{r}}$. Hence, by the Arzelá-Ascoli theorem, $\mathscr{G}_{1}$ is compact on $\bar{B}_{\bar{r}}$.

(iii) From $\left(A_{1}\right)$ and (33) it follows that $\mathscr{G}_{2}$ is a contraction mapping.

Thus all the conditions of Lemma 13 are satisfied. Hence, by the conclusion of Lemma 13, the problem (2) has at least one solution on $[0,1]$.

As a special case, for $\chi(x) \equiv 1$, there always exists a positive $\bar{r}$ such that (34) holds true. In consequence, we have the following corollary.

Corollary 15. Let $f:[0,1] \times \mathbb{R} \rightarrow \mathbb{R}$ be a continuous function satisfying $\left(A_{1}\right)$. Furthermore, $|f(t, x)| \leq \xi(t)$, $\forall(t, x) \in[0,1] \times \mathbb{R}$, and $\xi \in C\left([0,1], \mathbb{R}^{+}\right)$. Then the boundary value problem (2) has at least one solution on $[0,1]$ provided (33) holds.

\section{Examples}

Example 1. Consider the problem

$$
\begin{gathered}
{ }^{c} D_{q}^{3 / 2} x(t)+\frac{1}{4} x(t)=\frac{1}{5} x+\cos x, \quad 0 \leq t \leq 1, \\
x(0)=0, \quad x(1)=I_{q} x\left(\frac{1}{3}\right),
\end{gathered}
$$

where $q=1 / 2, \lambda=1 / 4, \eta=1 / 3$. It is easy to find that $\delta_{1}=$ $27 / 25$, and

$$
|f(t, x)|=\left|\frac{1}{5} x+\cos x\right| \leq \frac{1}{5}|x|+1 .
$$

Obviously $h_{1}=1 / 5, h_{2}=1, \vartheta(\omega)=\omega$. In consequence, $\nu_{1}=0.3002935758, v_{2}=1.501467879$, and condition (21) yields $\omega>4.629306104$. Thus, the hypothesis of Theorem 9 is satisfied. Hence it follows by the conclusion of Theorem 9 that there exists at least one solution for the problem (39).

Example 2. Consider the nonlocal boundary value problem given by

$$
\begin{gathered}
{ }^{c} D_{q}^{3 / 2} x(t)+\frac{1}{4} x(t)=L\left(\cos t+\tan ^{-1} x\right), \quad 0 \leq t \leq 1, \\
x(0)=0, \quad x(1)=I_{q} x\left(\frac{1}{3}\right) .
\end{gathered}
$$

Here $q=1 / 2, \lambda=1 / 4, \eta=1 / 3$ and $L$ is a constant to be fixed later on. With the given data, it is found that $\delta_{1}=$ $27 / 25,|f(t, x)-f(t, y)| \leq L|x-y|$ and

$$
\Lambda=\frac{1}{\Gamma_{1 / 2}(5 / 2)}\left(1+\frac{27 \sqrt{3}(4 \sqrt{2}-1)+6 \sqrt{2}}{25 \sqrt{3}(4 \sqrt{2}-1)}\right)\left(L+\frac{1}{4}\right) .
$$

Letting

$$
L<\left[\frac{1}{\Gamma_{1 / 2}(5 / 2)}\left(1+\frac{27 \sqrt{3}(4 \sqrt{2}-1)+6 \sqrt{2}}{25 \sqrt{3}(4 \sqrt{2}-1)}\right)\right]^{-1}-\frac{1}{4},
$$

all the conditions of Corollary 12 are satisfied. Therefore, the conclusion of Corollary 12 applies to the problem (41).

\section{Conflict of Interests}

The authors declare that there is no conflict of interests regarding the publication of this paper.

\section{Acknowledgments}

The authors thank the referees for their useful suggestions that led to the improvement of the original paper. This work has been partially supported by Deanship of Scientific Research (DSR), King Abdulaziz University, Jeddah, Saudi Arabia.

\section{References}

[1] A. A. Kilbas, H. M. Srivastava, and J. J. Trujillo, Theory and Applications of Fractional Differential Equations, vol. 204 of North-Holland Mathematics Studies, Elsevier Science B.V., Amsterdam, The Netherlands, 2006.

[2] I. Podlubny, Fractional Differential Equations, Academic Press, San Diego, Calif, USA, 1999. 
[3] J. Sabatier, O. P. Agrawal, and J. A. T. Machado, Eds., Advances in Fractional Calculus: Theoretical Developments and Applications in Physics and Engineering, Springer, Dordrecht, The Netherlands, 2007.

[4] S. G. Samko, A. A. Kilbas, and O. I. Marichev, Fractional Integrals and Derivatives, Theory and Applications, Gordon and Breach Science, Yverdon, Switzerland, 1993.

[5] D. Băleanu and O. G. Mustafa, "On the global existence of solutions to a class of fractional differential equations," Computers \& Mathematics with Applications, vol. 59, no. 5, pp. 1835-1841, 2010.

[6] D. Băleanu, O. G. Mustafa, and R. P. Agarwal, "On $L^{p}$-solutions for a class of sequential fractional differential equations," Applied Mathematics and Computation, vol. 218, no. 5, pp. 20742081, 2011.

[7] R. P. Agarwal and B. Ahmad, "Existence theory for anti-periodic boundary value problems of fractional differential equations and inclusions," Computers \& Mathematics with Applications, vol. 62, no. 3, pp. 1200-1214, 2011.

[8] B. Ahmad and J. J. Nieto, "Riemann-Liouville fractional integrodifferential equations with fractional nonlocal integral boundary conditions," Boundary Value Problems, vol. 2011, article 36, 2011.

[9] S. Bhalekar, V. Daftardar-Gejji, D. Baleanu, and R. Magin, "Fractional Bloch equation with delay," Computers \& Mathematics with Applications, vol. 61, no. 5, pp. 1355-1365, 2011.

[10] F. T. Akyildiz, H. Bellout, K. Vajravelu, and R. A. Van Gorder, "Existence results for third order nonlinear boundary value problems arising in nano boundary layer fluid flows over stretching surfaces," Nonlinear Analysis. Real World Applications, vol. 12, no. 6, pp. 2919-2930, 2011.

[11] B. Ahmad and J. J. Nieto, "Sequential fractional differential equations with three-point boundary conditions," Computers \& Mathematics with Applications, vol. 64, no. 10, pp. 3046-3052, 2012.

[12] J. R. Graef, L. Kong, and Q. Kong, "Application of the mixed monotone operator method to fractional boundary value problems," Fractional Differential Calculus, vol. 2, pp. 554-567, 2011.

[13] R. P. Agarwal, "Certain fractional $q$-integrals and $q$-derivatives," Proceedings of the Cambridge Philosophical Society, vol. 66, pp. 365-370, 1969.

[14] W. A. Al-Salam, "Some fractional q-integrals and q-derivatives," Proceedings of the Edinburgh Mathematical Society, vol. 15, pp. 135-140, 1966.

[15] F. M. Atici and P. W. Eloe, "Fractional q-calculus on a time scale," Journal of Nonlinear Mathematical Physics, vol. 14, no. 3, pp. 333-344, 2007.

[16] F. M. Atici and P. W. Eloe, "Linear systems of fractional nabla difference equations," The Rocky Mountain Journal of Mathematics, vol. 41, no. 2, pp. 353-370, 2011.

[17] F. M. Atıc1 and P. W. Eloe, "Two-point boundary value problems for finite fractional difference equations," Journal of Difference Equations and Applications, vol. 17, no. 4, pp. 445-456, 2011.

[18] M. El-Shahed and F. M. Al-Askar, "Positive solutions for boundary value problem of nonlinear fractional $q$-difference equation," ISRN Mathematical Analysis, vol. 2011, Article ID 385459, 12 pages, 2011.

[19] R. A. C. Ferreira, "Positive solutions for a class of boundary value problems with fractional q-differences," Computers \& Mathematics with Applications, vol. 61, no. 2, pp. 367-373, 2011.
[20] R. A. C. Ferreira, "Nontrivial solutions for fractional $q$-difference boundary value problems," Electronic Journal of Qualitative Theory of Differential Equations, vol. 70, pp. 1-10, 2010.

[21] C. S. Goodrich, "Existence and uniqueness of solutions to a fractional difference equation with nonlocal conditions," Computers \& Mathematics with Applications, vol. 61, no. 2, pp. 191-202, 2011.

[22] J. R. Graef and L. Kong, "Positive solutions for a class of higher order boundary value problems with fractional $q$-derivatives," Applied Mathematics and Computation, vol. 218, no. 19, pp. 9682-9689, 2012.

[23] J. Ma and J. Yang, "Existence of solutions for multi-point boundary value problem of fractional $q$-difference equation," Electronic Journal of Qualitative Theory of Differential Equations, vol. 92, pp. 1-10, 2011.

[24] G. C. Wu and D. Baleanu, "New applications of the variational iteration methodfrom differential equations to $q$-fractional difference equations," Advances in Difference Equations, vol. 2013, article 21, 2013.

[25] F. Jarad, T. Abdeljawad, and D. Baleanu, "Stability of $q$-fractional non-autonomous systems," Nonlinear Analysis. Real World Applications, vol. 14, no. 1, pp. 780-784, 2013.

[26] B. Ahmad, S. K. Ntouyas, and I. K. Purnaras, "Existence results for nonlocal boundary value problems of nonlinear fractional q-difference equations," Advances in Difference Equations, vol. 2012, article 140, 2012.

[27] P. M. Rajković, S. D. Marinković, and M. S. Stanković, "Fractional integrals and derivatives in q-calculus," Applicable Analysis and Discrete Mathematics, vol. 1, no. 1, pp. 311-323, 2007.

[28] M. H. Annaby and Z. S. Mansour, q-Fractional Calculus and Equations, vol. 2056 of Lecture Notes in Mathematics, Springer, Heidelberg, Germany, 2012.

[29] P. M. Rajković, S. D. Marinković, and M. S. Stanković, "On $q$-analogues of Caputo derivative and Mittag-Leffler function," Fractional Calculus \& Applied Analysis, vol. 10, no. 4, pp. 359373, 2007.

[30] A. Granas and J. Dugundji, Fixed Point Theory, Springer Monographs in Mathematics, Springer, New York, NY, USA, 2005.

[31] D. R. Smart, Fixed Point Theorems, Cambridge University Press, 1980. 


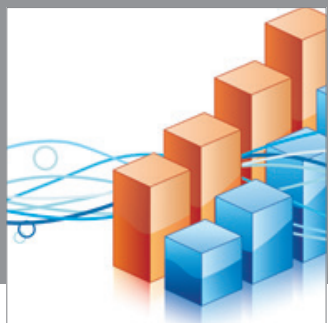

Advances in

Operations Research

mansans

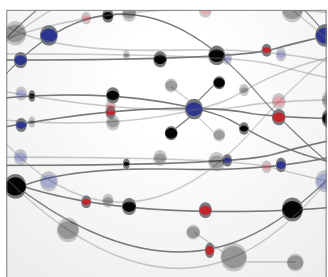

The Scientific World Journal
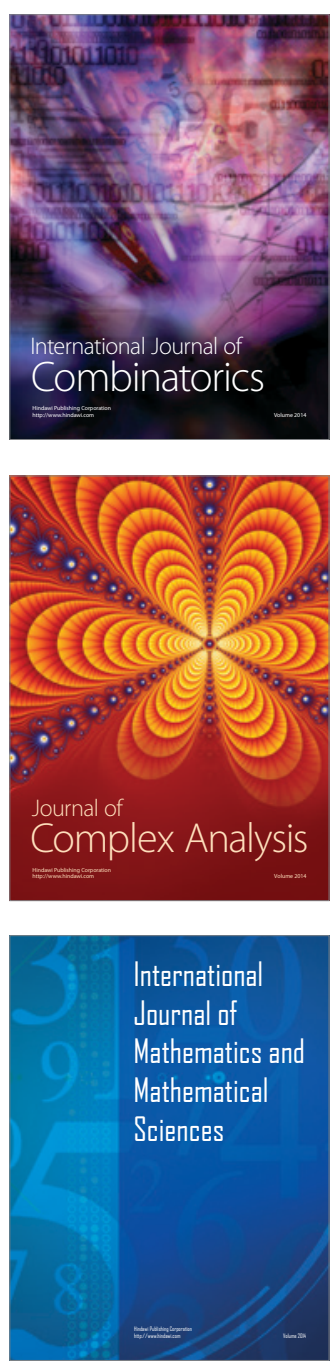
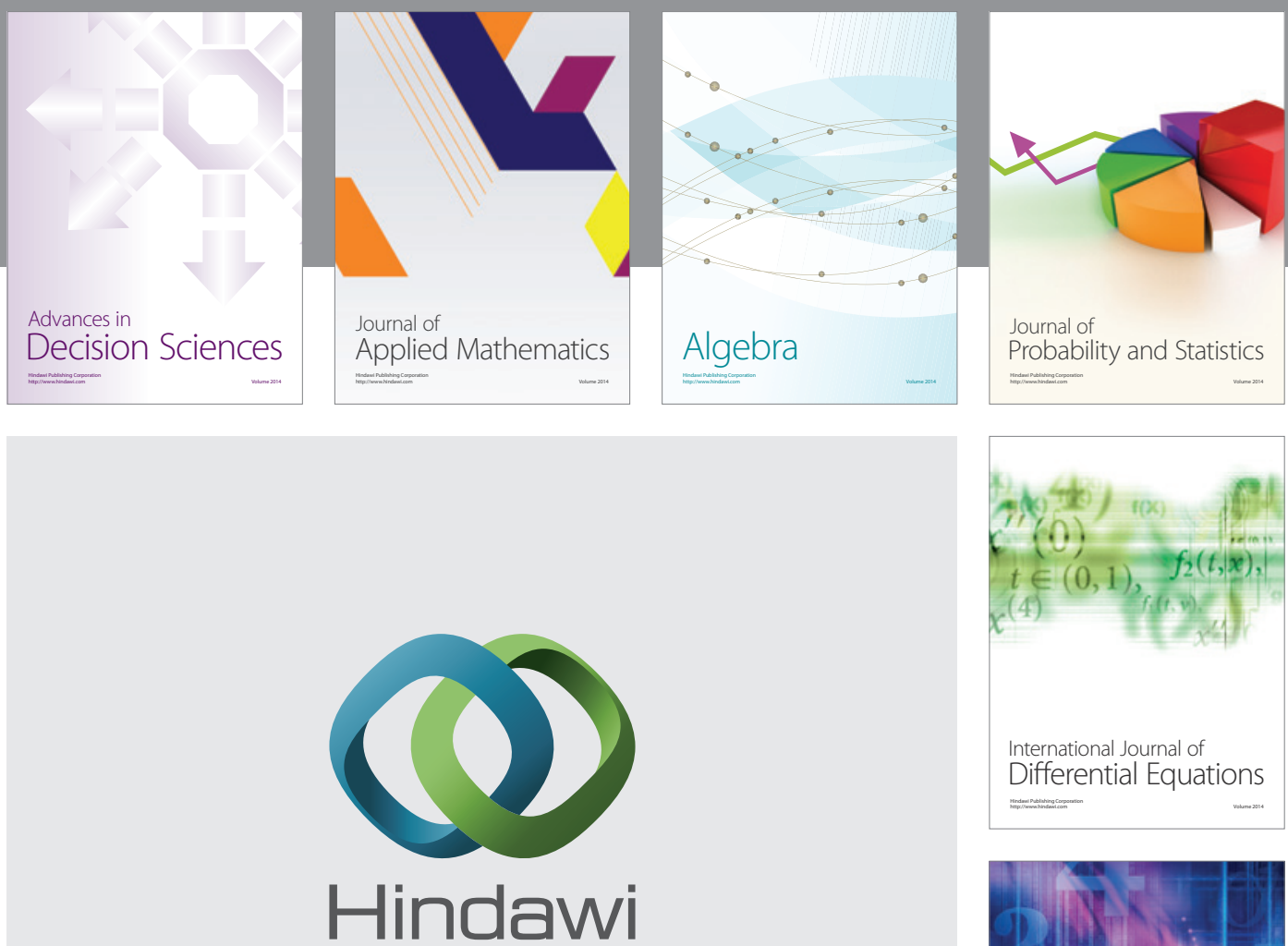

Submit your manuscripts at http://www.hindawi.com
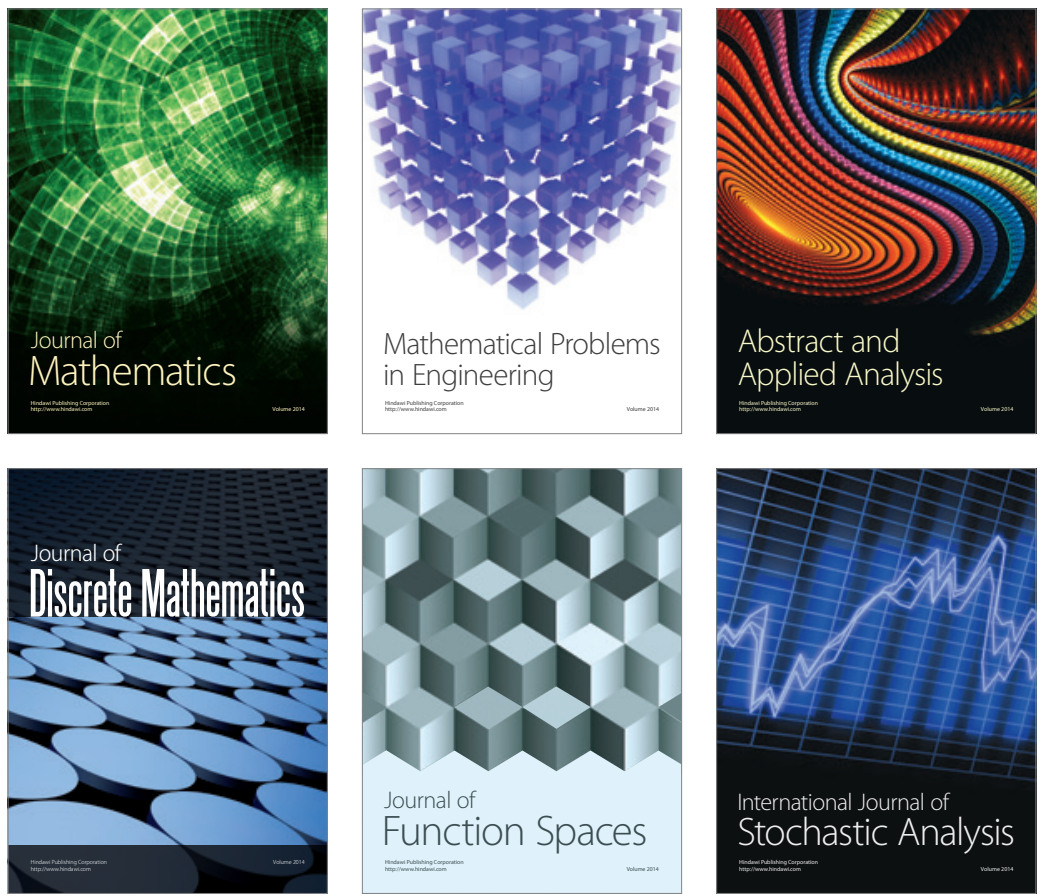

Journal of

Function Spaces

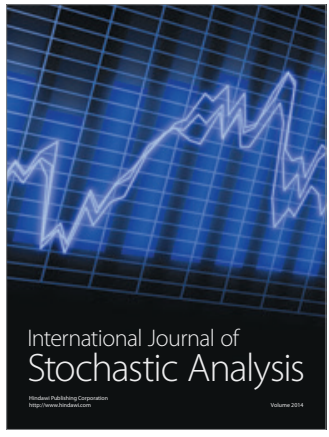

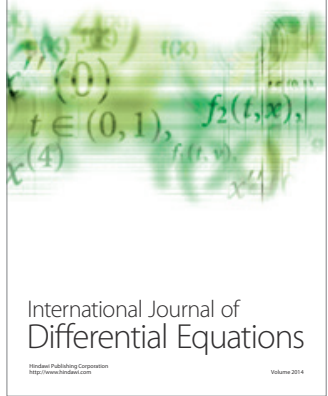
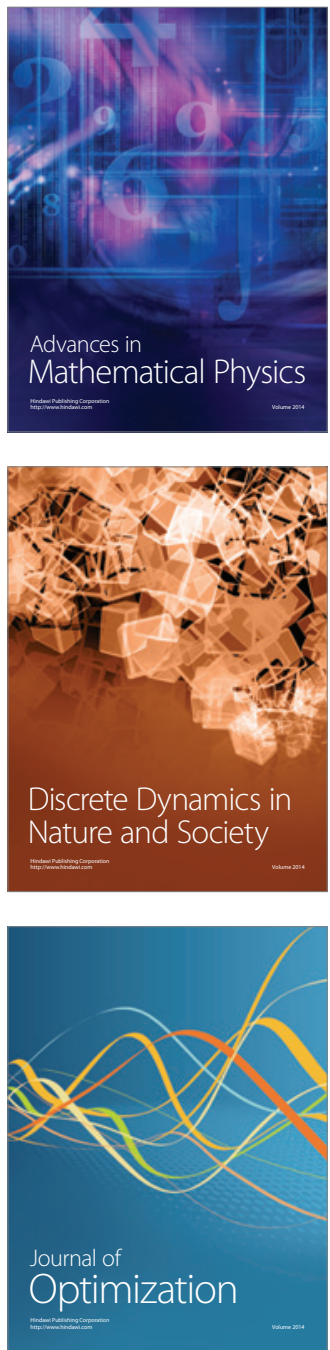